\title{
COLABORAÇAO NO DESIGN PARA A CUSTOMIZAÇÃO EM MASSA: SITUAÇÃO E PERSPECTIVAS
}

Isabela Mantovani Fontana

Universidade Federal do Paraná

isabelafontana@gmail.com

Adriano Heemann

Universidade Federal do Paraná

adriano.heemann@gmail.com

Resumo: $O$ presente artigo tem como objetivo apresentar as principais ênfases e lacunas teóricas sobre a colaboração no design para customização em massa (CM). Para tanto, o procedimento executado contempla uma revisão bibliográfica sistemática (RBS), que resultou em um mapeamento dos principais trabalhos publicados e disponíveis na base de dados Web of Science. Com esse mapeamento é possível sugerir que as publicações enfatizam alguns tipos específicos de colaboração dentro de algumas partes do processo a CM. Porém, identifica-se uma importante lacuna teórica também nesse aspecto, pois não foram encontrados, nesta amostra de artigos, estudos aprofundados de processos colaborativos entre os participantes do design para a CM e algumas especificidades nesse processo, 0 que acarreta em dificuldades para o avanço da CM no campo do design.

Palavras-chave: Design colaborativo, customização em massa, desenvolvimento de produto, revisão bibliográfica sistemática.

Resumo: This article aims to present the main emphases and theoretical gaps on collaboration in the design for mass customization (MC). Therefore, the procedure undertaken include a systematic literature review, which resulted in a mapping of the main works published and available at the Web of Science database. With this mapping it is possible to suggest that the publications emphasize specific types of collaboration within some parts of the process CM. However, it is identified an important theoretical gap also in this respect, since there were not found, in this sample of articles, in-depth studies of collaborative processes among the participants of design for the CM and some specifics in this process, which results in difficulties on the advances of CM in the design field.

Palavras-chave: Collaborative design, mass customization, product development, systematic literature review. 


\section{INTRODUÇÃO}

A crescente necessidade de mudanças nos sistemas produtivos a fim de sanar a atual demanda por velocidade e personalização dos produtos entregues aos consumidores fez com que o modelo de produção em massa e suas variações sofressem mudanças em sua concepção original com o intuito de se adaptar a essa nova realidade de mercado.

A customização em massa (CM) é um conceito emergente na indústria destinado a fornecer produtos customizados através de processos flexíveis em grandes volumes e com custos razoavelmente baixos (FRUTOS \& BORENSTEIN, 2004).

A essência da CM está na capacidade de desenvolvedores de produtos em perceber e captar nichos de mercado latentes e, posteriormente, desenvolver as capacidades técnicas para atender às diversas necessidades desse novo cliente alvo (JIAO et al., 2003).

É um desafio chegar a um consenso entre as várias partes envolvidas no desenvolvimento de produtos, considerando as diferentes responsabilidades e preocupações de cada um e decisões sobre desenvolvimento de produtos são cruciais, porém complexas. O processo de desenvolvimento de produtos é considerado como uma arma competitiva que ajuda as empresas a sobreviver e ter sucesso em mercados dinâmicos (CHAN \& IP, 2011).

Para alcançar essa vantagem competitiva, decisões sensatas devem ser feitas sobre os vários aspectos do desenvolvimento de produtos, tais como, por exemplo, os atributos do produto, o segmento de cliente e estratégias de marketing. Estas decisões estão interligadas e acabam por afetar a rentabilidade final do produto (CHAN \& IP, 2011).

Então, de um modo geral, a CM individualiza bens e serviços do mercado de massa para satisfazer as necessidades específicas de um cliente (LUO et al., 2008). Nesse sistema de produção, consumidores, fabricantes e fornecedores estão ligados em toda a operação. Materiais, peças, montagens, informações e ideias fluem através da rede de relacionamentos (LUO et al., 2008).

Uma grande parte do conhecimento necessário para a especificação do produto tem características tácitas, que precisam ser formalmente adquiridas para serem corretamente transformadas em um produto ou serviço que um consumidor específico requer.

O design voltado para a produção através da manufatura tradicional dispõe de metodologias e ferramentas consolidadas, assim como estudos que abordam a colaboração entre os atores desse processo. Porém, o processo tradicional de DNP também parece apresentar limitações sobretudo no que diz respeito a demandas operacionais observadas no de CM.

Portanto, parece existir um desafio emergente no que tange a prática do design para a CM, especificamente no que tange a interação entre os participantes do processo, a fim de alcançar uma melhor eficiência operacional.

Dada a oportunidade de facilitar esse processo de design para a CM intensificando a integração e colaboração entre as áreas participantes e utilizando ferramentas adequadas à esse tipo de processo, a presente investigação parte do seguinte questionamento: "Qual é a situação atual da pesquisa sobre colaboração no processo de design para a CM? ", a fim de identificar principais ênfases e possíveis lacunas no que tange essa importante prática para a atualidade. 
Para tanto, seguiu-se uma ordem de procedimentos que estruturam este artigo que se inicia com a apresentação geral do problema, seguindo pelo detalhamento do método utilizado. A partir dos resultados obtidos, foi feita uma análise que permitiu elaborar considerações finais e possíveis desenvolvimentos futuros desta pesquisa.

\section{MÉTODO}

Conforme já descrito anteriormente, a presente investigação busca determinar a situação atual da pesquisa em colaboração no design voltado para a $\mathrm{CM}$, identificando principais ênfases e possíveis lacunas. Não há, contudo, a intenção de exaurir o conhecimento teórico a esse respeito. A motivação aqui enfocada emerge da possível interligação entre os conceitos de colaboração no design e o desenvolvimento de produtos para a $\mathrm{CM}$, considerando a existência de estudos anteriores nesse âmbito embora ainda no que tangem produtos projetados para formas tradicionais de produção.

Para tanto, foi executada uma revisão bibliográfica sistemática (RBS) com base no procedimento iterativo de processamento de dados de Conforto et al. (2011): fase 1 de "entrada" (definição do problema, objetivos, fontes primárias, strings de busca, critérios de inclusão, critérios de qualificação, métodos e ferramentas, e cronograma), fase 2 de "processamento" (condução das buscas, análise dos resultados, documentação), e fase 3 de "saída" (alertas, cadastro e arquivo, síntese de resultados, e modelos teóricos).

O banco de dados utilizado foi o da Thomson Reuters, Web of Science por sua abrangência de áreas de pesquisa e periódicos internacionais indexados e a busca foi feita utilizando os três conceitos principais desta pesquisa: customização em massa, design e colaboração.

Essa busca foi feita utilizando os termos, em inglês, "mass customization", sua variação "mass customisation", "design", juntamente com o termo "collaboration". Esta busca teve como objetivo identificar publicações que englobem como tópicos processos colaborativos de desenvolvimento de produtos para a CM, indicando o intervalo de tempo desde o primeiro trabalho encontrado que atenda à essas especificações, até o ano de 2016. Esta busca resultou em 42 publicações que, dentre essas, somente 13 estão disponíveis na forma de artigo completo digital na base de dados utilizada.

Seguiu-se então a para a análise de conteúdo dos artigos completos resultantes para a identificação de características específicas dos assuntos e processos que abordam. Foi estipulado um escopo de conceitos para que esses artigos fossem mantidos como pertinentes para a presente pesquisa: trabalhos que tratem de processos de design de produtos ou serviços para a CM e que utilizem algum tipo de colaboração entre os participantes desse processo.

A análise dos artigos resultou em um mapeamento que permitiu identificar quais são as principais ênfases nas pesquisas atuais encontradas e quais as lacunas que este apanhado de artigos possui quanto ao design colaborativo para a CM.

A RBS adotada proporcionou resultados, que são detalhados na próxima seção e que permitem uma análise que precede o mapeamento da situação atual da pesquisa sobre colaboração no design voltado para a CM.

\section{RESULTADOS}

A RBS resulta em uma lista com 42 publicações que, dentre essas, 13 estão disponíveis na forma de artigo completo digital na base de dados utilizada. 
Conforme Tabela 1, os artigos disponíveis que contemplam os três tópicos utilizados na busca - "mass customization" ou "mass cutomisation", "design" e "collaboration" - foram publicados entre os anos de 2004 e 2015 e a maioria aborda a CM no contexto do produto físico.

\begin{tabular}{|c|c|c|c|c|}
\hline & AUTORES & ANO & CONTEXTO DA CM & CONTEXTO DA COLABORAÇÃO \\
\hline $\mathbf{1}$ & Frutos et al. & 2004 & Produto/Serviço & Designers e consumidores \\
\hline $\mathbf{2}$ & Matsumoto et al. & 2006 & Produto & Fabricantes e fornecedores \\
\hline $\mathbf{3}$ & Howard \& Squire & 2007 & Produto & Fabricantes e fornecedores \\
\hline $\mathbf{4}$ & Mikkola & 2007 & Produto & Participantes do sistema de manufatura \\
\hline $\mathbf{5}$ & Ming et al. & 2007 & Produto & Participantes da cadeia de suprimentos \\
\hline $\mathbf{6}$ & Trappey \& Hsiao & 2008 & Produto & Designers e tomadores de decisão \\
\hline $\mathbf{7}$ & Zha et al. & 2008 & Produto & Fonsumidores, gestores, designers, fabricantes e \\
\hline $\mathbf{8}$ & Hren \& Jezernik & 2009 & Produto & Fabricantes e fornecedores \\
\hline $\mathbf{9}$ & Squire et al. & 2009 & Produto & Funcionários de TI com participantes da CM \\
\hline $\mathbf{1 0}$ & Peng et al. & 2011 & Produto & Cadeia de suprimentos e consumidores \\
\hline $\mathbf{1 1}$ & Roh et al. & 2014 & Produto & Arquitetos e engenheiros \\
\hline $\mathbf{1 2}$ & Altan et al. & 2015 & Produto & Pesquisadores de áreas diversas \\
\hline $\mathbf{1 3}$ & Williams et al. & 2015 & Produto & \\
\hline
\end{tabular}

Tabela 1 - Características gerais da amostra de artigos encontrada (Fonte: Autores)

Os artigos também abordam a colaboração em perspectivas diferentes, conforme a coluna de "Contexto da Colaboração" também lista a Tabela 1. O primeiro artigo (Frutos et al., 2004) propõe um sistema de apoio à decisão para facilitar a criação e colaboração do cliente no processo de seleção de configuração do produto para a CM. O sistema integra programação orientada a objetos, análise de decisão multiatributo e programação linear inteira para apoiar a personalização do produto ou serviço. A escolha do cliente é gerida pela relevância relativa de um conjunto de atributos, bem como um conjunto de combinações de componentes oferecidos pela empresa, contido por um conjunto de limitações técnicas, estéticas e financeiras definidas de forma interativa por designers e clientes. Esse sistema é aplicado para personalização apartamentos residenciais, pois os autores consideram que um apartamento residencial pode é um dos produtos mais customizáveis, uma vez que há uma enorme quantidade de componentes que configuram o produto.

Já, o artigo de Matsumoto et al. (2006), aborda a colaboração na CM no âmbito da otimização total do processo. Os autores afirmam que a CM é um processo pelo qual são produzidos pequenos lotes de peças ou produtos individualizados que proporcionam a cada cliente algo individual de acordo com suas necessidades específicas. Neste artigo é examinada a cadeia de abastecimento entre o fabricante e fornecedor na indústria automobilística. Uma estratégia de colaboração é proposta nesse ambiente de CM seguindo preceitos de otimização total na cadeira de suprimentos a fim de diminuir custos, controlar o prazo de entrega de fornecedores e fabricantes assim como manter a qualidade esperada.

Howard \& Squire (2007) examinam o impacto da modularização do produto na gestão de relacionamento com fornecedores e identificam mais especificamente as condições de mediação que levam à colaboração entre empresas de compradores e fornecedores envolvidos em nessa troca modular que está presente na produção voltada para a CM. Este artigo fornece evidência empírica que mostra que a modularização do 
produto leva a uma maior colaboração por causa dos fatores mediadores presentes nesta troca. Os autores relatam uma survey com informações sobre as relações com os fornecedores de 500 unidades de produção no Reino Unido. Essa amostra de empresas utilizada pelos autores é apresentada a partir do banco de dados Conquest Business Media.

Já Mikkola (2007), investiga a gestão de arquitetura de produto modular para a CM. O artigo explica que esse modelo de gestão fornece uma maneira sistemática de medir o grau de modularidade incorporado em arquiteturas de produtos, que permite gestores e pesquisadores comparar diferentes conjuntos de arquiteturas do produto sem subjetividade. Esse modelo é testado nesta pesquisa através de um sistema hipotético.

No artigo de Ming et al. (2007), a CM é descrita como uma das tecnologias-chave na gestão do ciclo de vida do produto para fornecer produtos adaptados aos clientes finais com o custo de produção em massa. Os autores afirmam que a CM é caracterizada por produção com lead-time curto, requisitos dinâmicos do consumidor, tamanho pequeno de lotes e com alta variedade. O estudo sugere uma nova tecnologia com função modular de design para a colaboração na fabricação visando satisfazer as necessidades de produção em massa com baixo custo na indústria de ferramental. Com base nesse modelo de recurso modular, um projeto integrado para fabricação de sistema de colaboração é implementado para produção em massa de famílias de produtos de ferramental, que por causa de sua complexidade, alta variedade e lotes pequenos, se encaixam nos preceitos da CM.

Trappey \& Hsiao (2008) escolhem utilizar a perspectiva da colaboração entre participantes da cadeia de suprimentos. Os autores descrevem, uma vez que o tempo de inserção de um produto no mercado vem diminuindo, a CM está se tornando uma tendência no mercado orientada para o consumidor. Para alcançar os requisitos de mercado, torna-se importante para uma empresa ajustar o design de produto e seus processos de engenharia, sempre que as exigências do mercado mudam. Quando uma alteração de design ocorre, toda a cadeia de suprimentos é afetada. Seguindo o raciocínio dos autores, quando a informação correspondente à essas alterações não são eficientemente compartilhada entre os participantes na cadeia de abastecimento, é possível que ocorra um grande desperdício de tempo e recursos para a produção e logística. $\mathrm{O}$ artigo ainda descreve o desenvolvimento e aplicação na indústria automotiva de uma plataforma denominada Advanced Production Quality Planning (APQP) que proporciona, principalmente, um ambiente colaborativo que reforça a visibilidade das operações da cadeia de suprimentos. Essa plataforma também contribui para a troca e arquivamento de documentos entre os parceiros da cadeia, de modo a garantir a qualidade da produção em conformidade com o padrão da indústria.

O sétimo artigo listado na Tabela 1, Zha et al., (2008), sugere que o design de sistemas complexos de engenharia tem se tornado cada vez mais uma tarefa de colaboração entre designers ou equipes de projeto que estão fisicamente, geograficamente e temporalmente distribuídos. Os autores descrevem como o design colaborativo envolve essencialmente os processos de tomada de decisão que requerem avaliação, comparação e seleção de alternativas de design, bem como eventual otimização de um ponto de vista sistemático. Eles afirmam, ainda, que aumentar o conhecimento de design e apoiar a colaboração entre os designers e outros tomadores de decisão (por exemplo, clientes) para tomar decisões inteligentes dentro de um processo de design, pode aumentar a eficiência do projeto e resultar em projetos de maior 
qualidade. Este artigo apresenta um modelo de decisão híbrido e um quadro multiagente de apoio à decisão no design colaborativo em dois tipos de produtos que possuem características da CM (motores universais e fontes de alimentação elétricas).

Seguindo, Hren \& Jezernik (2009) afirmam que a CM exige uma cadeia de colaboração. Essa cadeia colaborativa inclui os consumidores, gerentes, designers, fabricantes e fornecedores que devem trocar de soluções e informações, normalmente geograficamente de locais diferentes. Para suportar essa colaboração, os autores combinam o sistema de modelagem com protótipo virtual via Web para avaliação de produtos, envolvendo simultaneamente suas simulações de comportamento e testa sua abordagem em um caso real de controle remoto de portões.

Por outro lado, Squire et al. (2009) mostram a importância de compreender as capacidades e recursos dos fornecedores pressuposto para o desenvolvimento de colaborações estreitas. O artigo utiliza uma survey sobre a relação entre empresafornecedor para a coleta os dados. Os respondentes puderam escolher um relacionamento que era "crítico" para organização. Os respondentes foram selecionados por função de trabalho (gerente de compras ou equivalente), tamanho da planta de produção da empresa (pelo menos 50 trabalhadores) e setor da indústria (máquinas e outros equipamentos; máquinas de escritório e computadores; máquinas e aparelhos eléctricos; aparelhos médicos, instrumentos de precisão e ópticos, relógios; veículos automóveis, reboques e semirreboques; e outros equipamentos de transporte).

Peng et al. (2011) desenvolvem, com base na literatura existente, um quadro conceitual que relaciona vários tipos de tecnologias da informação (TI) com os recursos necessários para a CM. Os autores identificam quatro tipos de TI que estão associados com as capacidade e desempenho de CM. Essas relações são testadas usando dados recolhidos a partir de uma amostra de plantas de produção que fabricam uma ampla variedade de produtos customizados. Essas empresas estão localizadas em países desenvolvidos e produzem eletrônicos, máquinas e componentes para transporte.

Roh et al. (2014) argumentam que o sucesso das atividades de fabricação global muitas vezes depende da flexibilidade de uma empresa em termos de sua capacidade de adaptar a sua cadeia de suprimento às mudanças dinâmicas nas necessidades e preferências dos seus consumidores. Assim, os autores desenvolvem o artigo como uma estratégia de cadeia de suprimentos responsiva influencia o nível de compartilhamento de informações com os consumidores finais, no desenvolvimento de práticas de colaboração com fornecedores, na utilização de tecnologias de fabricação avançada e nas estratégias de produção, que tem sido empregadas como uma maneira para melhorar a eficiência de produção. A evidência empírica deste artigo foi desenvolvida a partir da International Manufacturing Strategy Survey (IMSS).

Altan et al. (2015) apresentam a remodelação de unidades habitacionais flexíveis e geminadas e sua avaliação de desempenho, utilizando um método de simulação de construção. O objetivo dos autores é planejar um conjunto de casas geminadas que, não só podem utilizar os conceitos de design flexível no layout de planejamento, mas também podem reduzir o consumo de energia com um design passivo com especial atenção à seleção de materiais. Esse tipo de habitação pode ajustar-se às necessidades dos diferentes inquilinos com a finalidade de alcançar uma construção sustentável sob diferentes aspectos, tais como baixo consumo de energia, uso de energias renováveis e materiais de baixo impacto, com design flexível. Os autores descrevem como a colaboração entre arquitetos e engenheiros (ou seja, auxiliando a concepção de uma 
unidade de habitação social sustentável) ajuda a encontrar soluções de conforto interior, combinando características estruturais e arquitetônicas com a integração de sistemas de energia, onde a tarefa de simulação de construção desempenhou um papel importante nesta colaboração.

Por fim, o artigo de Williams et al. (2015) explora, com base em resultados experiências de experiências anteriores no mesmo projeto, se superfícies hiperbólicas podem ser utilizadas para criar uma sala de reuniões que satisfaça requisitos acústicos e de conforto auditivos, chamadas de FabPod. Os autores explicam que a natureza colaborativa do projeto (conduzido entre diversos pesquisadores de diversas áreas de conhecimento) e interdependências entre os aspectos do projeto levou à concepção de um sistema de design genérico. Para tanto, são utilizadas uma série de ferramentas digitais para gerenciar a geometria do projeto e informações relacionados a ele, assim como processos manuais sobre material, montagem e controle de qualidade.

Para que seja possível identificar se os resultados enumerados e descritos permitem a identificação das principais ênfases ou possíveis lacunas no que tange a colaboração no design para a CM, uma análise crítica foi efetuada. Esta análise é descrita a seguir, indicando como os resultados foram analisados para alcançar o objetivo geral desta pesquisa.

\section{DISCUSSÃO}

Observa-se que com o advento da CM houve um aumento de interação entre os participantes do processo de design de produtos. A forma como o conhecimento relacionado à configuração de produtos é compartilhada entre os participantes desse processo parece determinar o sucesso desse tipo de sistema produtivo (FRUTOS et al., 2004).

Este processo é basicamente dependente de um fluxo eficiente de informações através de todos os agentes envolvidos, e em como as necessidades dos clientes, preferências e restrições são coletados, armazenados e processados.

A partir dos resultados bibliográficos obtidos e descritos, é possível sugerir inicialmente que a maior parte das publicações focam em processos colaborativos no design de produtos para a CM (Figura 1).

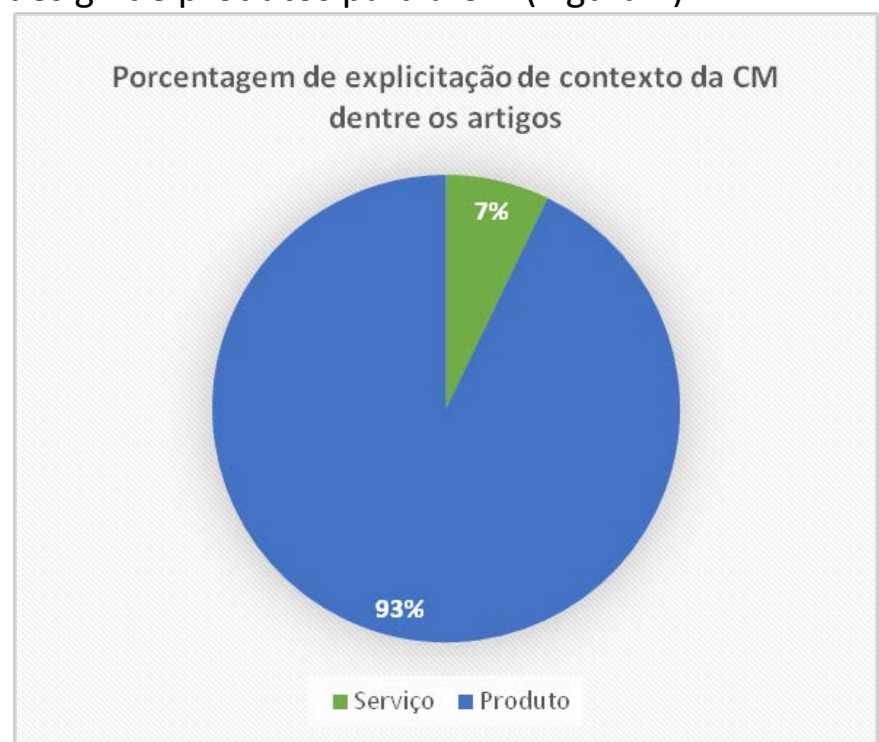

Figura 1 - Porcentagem que o tipo de contexto da CM é explicitado nos artigos analisados Fonte: Autores 
Apesar de um artigo (FRUTOS et al., 2004) citar que o seu sistema de suporte de decisões tem foco no desenvolvimento de produtos e serviços, porém somente explicita o "serviço" como uma possível aplicação, não fazendo parte do exemplo dado no artigo.

Já, no que tange o contexto em que a colaboração ocorre, existe uma variação de 12 tipos de relações colaborativas (listadas na Tabela 1 na coluna de "Contexto da Colaboração"). Dentre os participantes dessas relações, são agrupados e identificados 8 tipos participantes principais (Figura 2) para facilitar a divisão da frequência em que aparecem nos estudos, já que "participantes do processo de manufatura" englobam alguns dos participantes de outras relações.

\begin{tabular}{|c|}
\hline Participantes Identificados \\
Designers \\
\hline Consumidores \\
\hline Fabricantes \\
\hline Fornecedores \\
\hline Gestores \\
\hline Pesquisadores \\
\hline Participantes do sistema de manufatura \\
\hline Participantes da cadeia de suprimentos \\
\hline Tomadores de decisões \\
\hline Funcionários de TI \\
\hline Participantes da CM \\
\hline Cadeia de Suprimentos \\
\hline Arquitetos \\
\hline Engenheiros \\
\hline
\end{tabular}

\begin{tabular}{|c|c|} 
Participantes Principais \\
\hline \multirow{4}{*}{ Designers } \\
\cline { 2 - 2 } & Consumidores \\
\hline Fabricantes \\
\hline Fornecedores \\
\hline Gestores \\
\hline Pesquisadores \\
\hline Engenheiros \\
\hline Arquitetos \\
\hline
\end{tabular}

Figura 2 - Agrupamento dos participantes identificados nas colaborações descritas nos artigos analisados. Fonte: Autores

Na Figura 3, após o agrupamento dos participantes da colaboração, é possível identificar que quase a metade das relações colaborativas que são abordadas nos artigos que contém pesquisas sobre a colaboração no design voltado para a CM, estão permeando os fabricantes e os fornecedores. Já os gestores aparecerem em $15 \%$ das relações colaborativas indicadas.

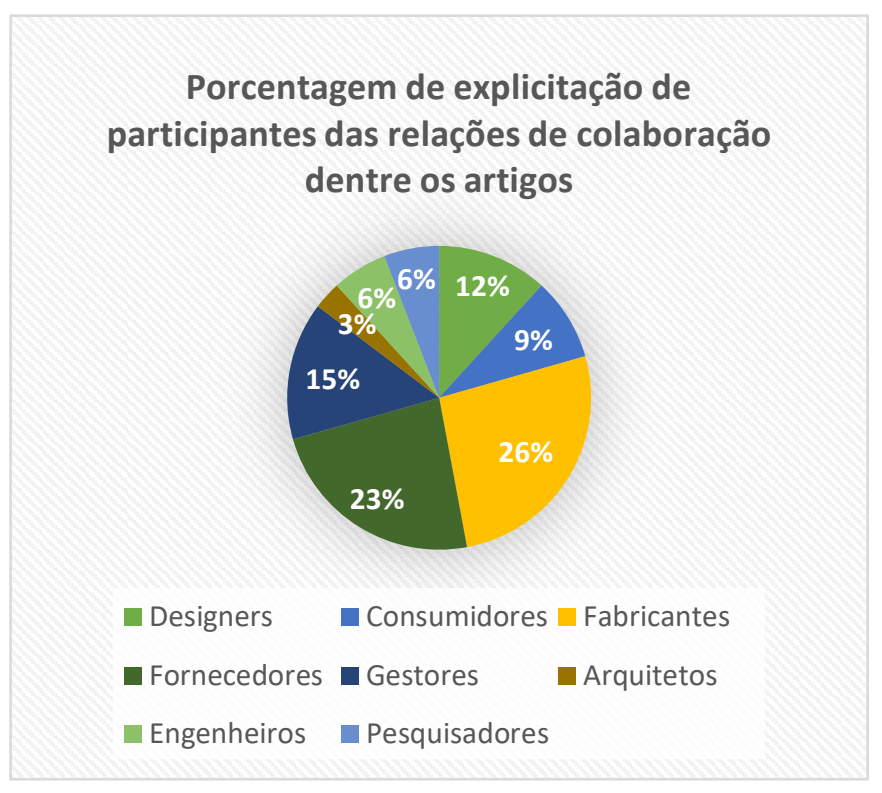

Figura 3 - Porcentagem dos participantes das colaborações identificadas nos artigos analisados Fonte: Autores 
Por tanto, identifica-se ênfases no conteúdo dos artigos analisados assim como lacunas teóricas no que tange a colaboração no design para a CM (Figura 4).

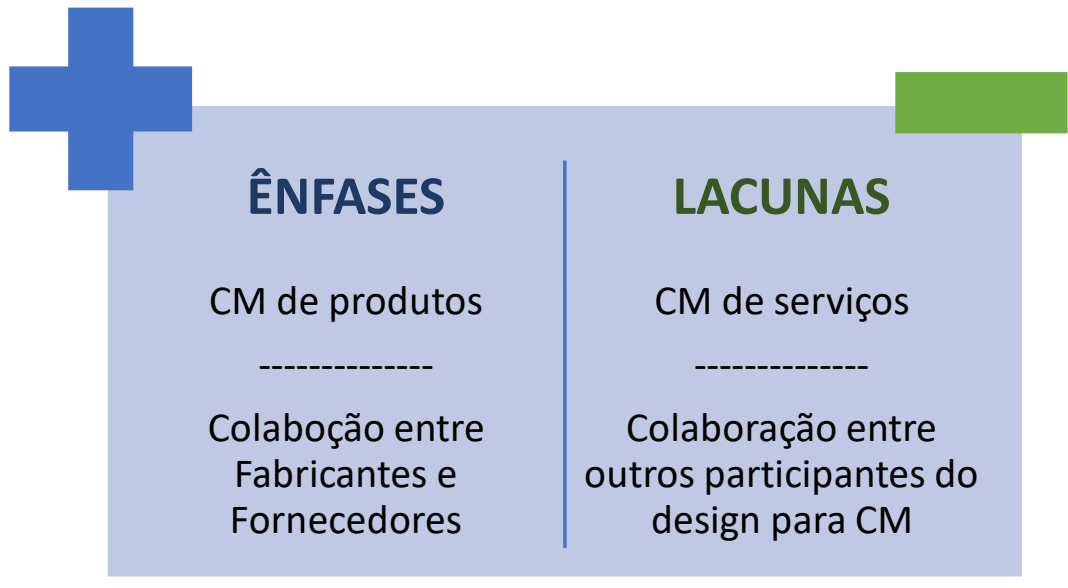

Figura 4 - Principais ênfases e lacunas identificadas nos artigos analisados Fonte: Autores

\section{CONCLUSÃO}

Partindo do objetivo de identificar a situação atual da pesquisa relacionada ao design colaborativo voltado para a $\mathrm{CM}$, o artigo seguiu etapas que permitiram a identificação das principais ênfases e lacunas dos artigos analisados nesta pesquisa. Após análise crítica, o texto apresentou que as principais ênfases são processos de design para $\mathrm{CM}$ de produtos e com relações colaborativas entre fabricantes e fornecedores. Já, as lacunas teóricas estão relacionadas a processos de design para CM de serviços e relações colaborativas entre os demais participantes do design para a CM.

Sugere-se, para desenvolvimento futuro, tornar a pesquisa mais abrangente, inserindo na análise conceitos específicos isolados de design colaborativo e design para CM na tentativa de identificar uma possível intersecção entre os dois assuntos e incluindo a literatura consolidada sobre esses conceitos em separado, que não foram identificadas na busca dos termos em conjunto.

\section{REFERÊNCIAS}

ALTAN, H.; GASPERINI, N.; MOSHAVER, S.; FREATTARI, A. Redesigning Terraced Social Housing in the UK for Flexibility Using Building Energy Simulation with Consideration of Passive Design. Sustainability (2015) 7, 5488-5507.

CHAN, S. L.; IP, W. H. A dynamic decision support system to predict the value of customer for new product development. Decision Support Systems 52 (2011) 178-188.

CONFORTO, E. C.; AMARAL, D. C.; SILVA, S. L. Roteiro para revisão bibliográfica sistemárica: aplicação no desenvolvimento de produtos e gerenciamento de projetos. 8으 Congresso Brasileiro de Gestão de Desenvolvimento de Produto (CBGDP 2011). Porto Alegre, RS - Brasil, 2011.

FRUTOS, J. D.; BORENSTEIN, D. A framework to support customer-company interaction in mass customization environments. Computers in Industry 54 (2004) 115-135. 
FRUTOS, J. D.; SANTOS, E. R.; BORESTEIN, D. (2004). Decision Support System for Product Configuration in Mass Customization Environments. Concurrent engineering: Research and Applications (2004).

HOWARD, M.; SQUIRE, B. Modularization and the impact on supply relationships. International Journal of Operations \& Production Management Vol. 27 Iss 11 pp. 1192 1212 (2007).

HREN, G.; JEZERNIK, A. A framework for collaborative product review. International Journal of Advanced Manufacturing Technology (2009) 42:822-830.

JIAO, J. X.; MA, Q. H.; TSENG, M. M. Towards high value-added products and services: mass customization and beyond. Technovation, v. 23, n. 10, p. 809-821, Oct 2003.

LUO, T.; XIONG, Z.; FANG, Y. A Framework of Knowledge Management for Mass Customization Internet-based. 2008 International Symposium on Information Science and Engineering.

MATSUMOTO, S.; UENO, N.; OKUHARA, K.; ISHII, H. Proposal of load leveling model for implementing mass customization in automobile industry. Journal of Advanced Mechanical Design, Systems, and Manufacturing, 06-0151 (2006)

MIKKOLA, J. H. Management of Product Architecture Modularity for Mass Customization: Modeling and Theoretical Considerations. IEEE Transactions on Engineering Management, vol. 54, no. 1 (2007).

MING, X. G.; YAN, J. Q.; LU, W. F.; MA, D. Z.; SONG, B. Mass production of tooling product families via modular feature-based design to manufacturing collaboration in PLM. J Intell Manuf (2007) 18:185-195.

PENG, D. X.; LIU, G. J.; HEIM, G. R. Impacts of information technology on mass customization capability of manufacturing plants. International Journal of Operations \& Production Management, Vol. 31 Iss 10 pp. 1022 - 1047 (2011).

$\mathrm{ROH}$, J.; HONG, P.; MIN, H. Implementation of a responsive supply chain strategy in global complexity: the case of manufacturing firms. Int. J. Production Economics 147 (2014) 198210.

SQUIRE, B.; COUSINS, P. D.; LAWSON, B.; BROWN, S. The effect of supplier manufacturing capabilities on buyer responsiveness. International Journal of Operations \& Production Management, Vol. 29 Iss 8 pp. 766 - 788 (2009).

TRAPPEY, A. J. C.; HSIAO, D. W. Applying collaborative design and modularized assembly for automotive ODM supply chain integration. Computers in Industry 59 (2008) 277-287.

WILLIAMS, N.; BURRY, J.; DAVIS, D.; BRASY, P.; LEON, A. P.; BURRY, M. FabPod: Designing with temporal flexibility \& relationships to mass-customisation. Automation in Construction 51 (2015) 124-131.

ZHA, X. F.; SRIRAM, R. D. FERNANDEZ, M. G.; MISTREE, F. Knowledge-intensive collaborative decision support for design processes: a hybrid decision support model and agente. Computers in Industry 59 (2008) 905-922. 